\title{
Neurological complications in HIV
}

\author{
Celia Hogan and Ed Wilkins
}

\section{Summary}

HIV is neuroinvasive with early involvement of the nervous system and has the potential to cause disease at any site of the neuro-axis during the evolution from seroconversion to late stage HIV. Disease may result from direct viral infection, indirect immune-deficiency driven opportunistic infections, AIDS-defining cancers, antiretroviral (ARV) drug therapy, or less well elucidated associations, such as vascular events (Table 1). Recognition of each of these is paramount in the prevention or attenuation of long-term morbidity.

Though the epidemiology of neurological disease has altered substantially since the arrival of combination antiretroviral therapy (cART), with reduced incidence and improved survival, the spectrum of central nervous system (CNS) diseases has remained relatively unchanged. Despite available treatment options, mortality remains high and the morbidity significant. CNS diseases can result in long hospital stays, reduced quality of life and marked disability. The majority of disease occurs in the advanced stages of HIV infection where immunosuppression is the predominant influence. Diagnosis can prove challenging as presentation is often atypical and there can be significant neurological involvement with limited evidence of disease. Multiple aetiologies can co-exist and investigations may yield unexpected results, rendering interpretation diffi-

Table 1. Differential diagnosis of HIV-related disorders of the nervous system.

$\begin{array}{ll}\text { Presentation } & \text { Main causes } \\ \text { Space occupying lesion(s) } & \begin{array}{l}\text { Toxoplasmosis, primary CNS lymphoma, PML, TB, } \\ \text { Cryptococcus }\end{array} \\ \text { Cognitive impairment } & \begin{array}{l}\text { HIV neurocognitive disorder - HIV-associated } \\ \text { dementia, syphilis, PML, CMV }\end{array} \\ \text { Encephalitis } & \text { HIV, varicella-zoster virus, herpes simplex, syphilis } \\ \text { Meningitis } & \text { HIV seroconversion, Cryptococcus, TB, syphilis } \\ \text { Polyradiculitis } & \text { CMV, non-Hodgkin's lymphoma } \\ \text { Peripheral neuropathy } & \text { HIV, drugs (eg d4T, ddI) } \\ \text { Retinitis } & \text { CMV, toxoplasmosis, retinal necrosis, HIV, syphilis } \\ \text { Spastic paraparesis } & \text { HIV-vacuolar myelopathy, HIV seroconversion, } \\ & \begin{array}{l}\text { transverse myelitis from varicella zoster, herpes } \\ \text { simplex, human T-cell lymphotropic virus 1, or } \\ \text { syphilis }\end{array}\end{array}$

$\mathrm{CMV}$ = cytomegalovirus; $\mathrm{CNS}=$ central nervous system; $\mathrm{PML}=$ progressive multifocal leukoencephalopathy; $\mathrm{TB}=$ tuberculosis.

Celia Hogan, specialist registrar, infectious diseases; Ed Wilkins, consultant, infectious diseases

Department of Infectious Disease, North Manchester General Hospital cult. Paradoxically, cART may also alter the way CNS disease manifests and unmask opportunistic infections or cause clinical representation of the opportunistic infections, when it represents immune reconstitution syndrome (IRS). Clinical assessment, imaging (typically magnetic resonance imaging (MRI)) and cerebral spinal fluid (CSF) sampling remain the chief diagnostic tools. This conference summary reviews these differing aspects.

\section{Background}

Despite the advent of cART, neurological disease is still the presenting feature of HIV in 2-3\% (compared to $15 \%$ pre-cART). Around two-thirds result from opportunistic infection and HIVrelated malignancies, and represent late stage disease with profound immunodeficiency $(<100$ cells/l). Nevertheless, patterns of disease have changed since the emergence of cART with an $80-90 \%$ fall in the incidence of opportunistic infections-related disease and HIVrelated dementia. However, the prevalence of the latter has increased with the overall improved prognosis of HIV-infected persons. Moreover, since 2003 there has been no further fall reflecting the fact that new patients are still presenting late with CNS-related disease.

Primarily, the type and severity of disease depends on the CD4 count and the timing of presentation; seroconversion (when the CD4 count is usually high) and late stage disease (when the CD4 count will typically be below $100 \mathrm{c} / \mathrm{mm}^{3}$ ). Age, ethnicity, drug therapy and co-morbidities are also significant factors (Table 2).

\section{Directly HIV-related (Table 3)}

Primary infection (seroconversion) may present as a neurological illness, importantly aseptic meningitis, facial palsy, Guillain-Barré (GB) syndrome and transverse myelitis. If severe, cART treatment is advocated. Only $1-4 \%$ of patients will develop acute neurological disease at this time but recognition is essential to allow appropriate work-up and treatment. Aseptic meningitis is the most common and can also occur in early disease when it can be asymptomatic. It is indistinguishable from enteroviral or other lymphocytic meningitides (normal glucose, normal/slightly elevated protein) but may be associated with unilateral or bilateral facial palsy. HIV-RNA is positive on CSF examination. Its course is benign but in $1-3 \%$ may be severe when ARV treatment is recommended. GB syndrome is rare but HIV accounts for $10 \%$ of cases and therefore screening is essential. Investigation and treatment are identical to non-HIV-related disease.

More commonly, HIV-related pathology manifests as a chronic and slowly progressive disease process (peripheral neuropathy, dementia, neurocognitive disorder, myelopathy) when combination ARV has limited or no effect and where additional factors, including co-morbidities and age, play a part. 
Table 2. CD4-related presentation.

\begin{tabular}{|c|c|c|}
\hline $\begin{array}{l}\text { Seroconversion and/or } \\
\text { CD4 >500 }\end{array}$ & $\begin{array}{l}\text { CD4 } \\
>200-<500\end{array}$ & CD4 $<200$ \\
\hline Aseptic meningitis & TB meningitis & HIV-associated dementia \\
\hline Meningoencephalitis & $\begin{array}{l}\text { Guillain-Barré } \\
\text { syndrome }\end{array}$ & TB meningitis \\
\hline Bell's palsy & DSPN & Cryptococcal meningitis \\
\hline Guillain-Barré syndrome & Polymyositis & Toxoplasmosis \\
\hline Brachial neuritis & MND & PML \\
\hline \multirow[t]{8}{*}{ Transverse myelitis } & & Primary CNS lymphoma \\
\hline & & Nocardia brain abscess \\
\hline & & DSPN \\
\hline & & Vacuolar myelopathy \\
\hline & & CMV encephalitis \\
\hline & & Ischaemic CVA \\
\hline & & Herpes encephalitis \\
\hline & & $\mathrm{CMV}$ radiculopathy \\
\hline
\end{tabular}

$\mathrm{CMV}=$ cytomegalovirus; $\mathrm{CNS}=$ central nervous system; $\mathrm{CVA}=$ cerebrovascular accident; DSPN = distal symmetrical polyneuropathy; MND = mild neurocognitive disorder; $\mathrm{PML}=$ progressive multiofocal leukoencephalopathy; $\mathrm{TB}=$ tuberculosis.

\section{Table 3. Directly HIV related.}

$\begin{array}{ll}\text { Early acute } & \text { Late chronic } \\ \text { Aseptic meningitis } & \text { AIDS dementia } \\ \text { Encephalitis } & \text { HIV-associated neurocognitive disorder } \\ \text { Bell's palsy } & \text { Vacuolar myelopathy } \\ \text { AIDM } & \text { DSPN } \\ \text { Transverse myelitis } & \begin{array}{l}\text { Chronic inflammatory demyelinating } \\ \text { neuropathy }\end{array} \\ \text { Brachial neuritis } & \text { Diffuse infiltrative lymphocytosis syndrome } \\ \text { Cauda equina } & \text { Myopathy } \\ \text { syndrome } & \\ \text { Polymyositis } & \end{array}$

AIDM = acute inflammatory demyelination polyneuropathy; DSPN = distal symmetrical polyneuropathy.

HIV-associated neurocognitive disorders (HAND) is the umbrella term to describe neurodegenerative disease caused by chronic HIV infection. It covers a wide variety of disorders ranging from asymptomatic neurocognitive impairment (ANI), HIV-associated mild neurocognitive disorder (MND) to the most severe, HIV-associated dementia (HAD). ${ }^{1}$ New evidence is emerging to the extent of HAND, with incidences ranging from $29 \%$ to $69 \%$ in the HIV positive population. ${ }^{2}$ Several associations in the development of HAND have been described in the literature; viral-related factors, such as clade, uncontrolled viraemia and a high CNS viral load (compared to plasma), ${ }^{3}$ immune- related factors (current and nadir CD4 counts), and co-infection with hepatitis C. ${ }^{4}$

HIV-associated dementia (HAD) is the most severe form of HAND. Patients exhibit a significant decline in cognition along with major functional impairment, with a detrimental impact on activities of daily living. Patients can exhibit signs of spasticity and/or extrapyramidal signs. Imaging shows widespread atrophy and CSF levels of HIV-RNA are high. The diagnosis remains clinical and it is important to exclude other potentially reversible causes of dementia such as, cytomegalovirus (CMV) encephalitis, progressive multifocal leukoencephalopathy (PML), neurosyphilis, depression, substance abuse and other medical causes.

Since the emergence of cART, the incidence of HAD has halved. ${ }^{5}$ However, due to improved HIV survival rates, the HIV elderly population has grown, leading to an overall increase in the cumulative prevalence of HAD. Choice of cART remains controversial. The primary objective of cART is to suppress HIV replication within the CNS. The level of CNS penetration by ART has yet to be fully established. Several studies have investigated ART concentrations within the CSF. ${ }^{6}$ However, it remains unclear to what extent CSF concentration reflects overall parenchymal concentration and activity. The ideal combination of ART in HAND has not yet been established.

Vacuolar myelopathy is HIV-specific and can occur at any stage but is more likely when the CD4 is $<50 \mathrm{c} / \mathrm{mm}^{3}$. Presenting features are spastic paraparesis, unsteady gait, autonomic dysfunction, and loss of dorsal column sensory modalities. Diagnosis is by exclusion, importantly human T-lymphotropic virus type I (HTLV1). The condition is irreversible and treatment symptomatic.

Lastly, distal sensory peripheral neuropathy (DSPN) is an uncommon but debilitating complication of HIV. It is associated with advanced immunosuppression and there is an increased risk with HAD, older age and substance misuse. DSPN is characterised by painful paraesthesia of feet and soles, shooting leg pains, stocking-glove sensory loss, and decreased vibratory sense in ankles with absent or reduced ankle jerks. Distinction from ART-related peripheral neuropathy is difficult. cART may have some impact early on in DSPN but treatment is often symptomatic.

\section{Opportunistic infections (Table 4)}

Cerebral toxoplasmosis is caused by the protozoan Toxoplasma gondii. Primary infection occurs after the ingestion of infected cysts from cat faeces or consuming undercooked meat. Disease appears to occur almost exclusively because of reactivation of latent tissue cysts. ${ }^{7}$ Clinical disease rarely occurs in patients with CD4 $>200$ $\mathrm{c} / \mathrm{mm}^{3}$ and typically the CD4 count is $<100 \mathrm{c} / \mathrm{mm}^{3}$. Patients present with headache, fever, seizures, confusion and focal neurological deficits. Imaging demonstrates multiple ring enhancing lesions, with marked surrounding oedema. The lesions typically occur around the basal ganglia, brain stem and cortex.

Clinical diagnosis is based upon demonstration of typical lesions on an MRI scan in a patient not on co-trimoxazole prophylaxis and with a CD4 $<200 \mathrm{c} / \mathrm{mm}^{3}$. Serological tests have limited use, although in the context of negative IgG toxoplasma antibodies an alternative diagnosis is more likely. Toxoplasma polymerase chain 
reaction of the CSF has high sensitivity and specificity but lumbar puncture (LP) may be precluded because of midline/tentorial shift. The definitive diagnosis is detection of the organism in tissue. This, however, requires a stereotactic computed tomography-guided needle biopsy, an invasive procedure with high complication rates. Therefore patients with reported lesions on the scan are started on empirical treatment and are rescanned two to four weeks later to assess response. Brain biopsy is reserved for those where the diagnosis is in doubt, classically when empirical therapy has failed, when there is a reliable history of co-trimoxazole prophylaxis or when there is a single lesion or the CD4 is $>200$. The role of positron emission tomography scanning is currently under investigation. First line therapy is with pyrimethamine (given with folinic acid) and either sulphadiazine or clindamycin. Treatment duration is six weeks followed by secondary prophylaxis with co-trimoxazole. Steroids should be avoided if possible as they may mask the diagnosis of primary CNS lymphoma. cART should be optimised or initiated around two weeks after the acute phase of the illness: immune reconstitution syndrome (IRS) may occur in 5-10\% and can mimic initial presentation.

Cryptococcal meningitis is caused by infection with the yeast Cryptococcus neoformans. It commonly causes a sub-acute meningitis with symptoms of fever, headache, visual disturbances and confusion. Disease is caused by inhalation of the yeast and dissemination throughout the body. It can potentially affect every organ but predominantly involves the brain and lungs. Diagnosis is reliant on CSF sampling with a positive India ink stain (sensitivity 75-85\%), cryptococcal antigen (sensitivity 95\%) or positive fungal culture $(>95 \%) .{ }^{8}$ Raised intracranial pressure (ICP) is common and can lead to severe complications such as blindness, seizures and coma. At diagnosis, approximately $75 \%$ of patients will have a high CSF opening pressure $\left(>20 \mathrm{~cm} \mathrm{H}_{2} \mathrm{O}\right)$. In this incidence it is vital to reduce the pressure with repeated LP and drainage. In severe cases a lumbar drain or ventricularperitoneal (VP) shunt is required. First line treatment is with liposomal amphotericin and 5-flucytosine for

Table 4. HIV-related opportunistic infections.

$\begin{array}{ll}\text { Central } & \text { Peripheral } \\ \text { Cerebral toxoplasmosis } & \text { CMV radiculitis } \\ \text { Cryptococcal meningitis } & \text { VZV transverse myelitis } \\ \text { CMV encephalitis } & \text { TB myelitis } \\ & \text { Syphilitic myelitis } \\ \text { Herpes simplex encephalitis } & \\ \text { Varicella-zoster encephalitis } & \\ \text { PML } & \\ \text { Tuberculosis meningitis } & \\ \text { Tuberculoma } & \\ \text { Neurosyphilis } & \\ \text { Nocardiosis } & \\ \text { Aspergillosis } & \end{array}$

$\mathrm{CMV}=$ cytomegalovirus; PML = progressive multifocal leukoencephalopathy; $\mathrm{TB}=$ tuberculosis; VZV = varicella-zoster virus.

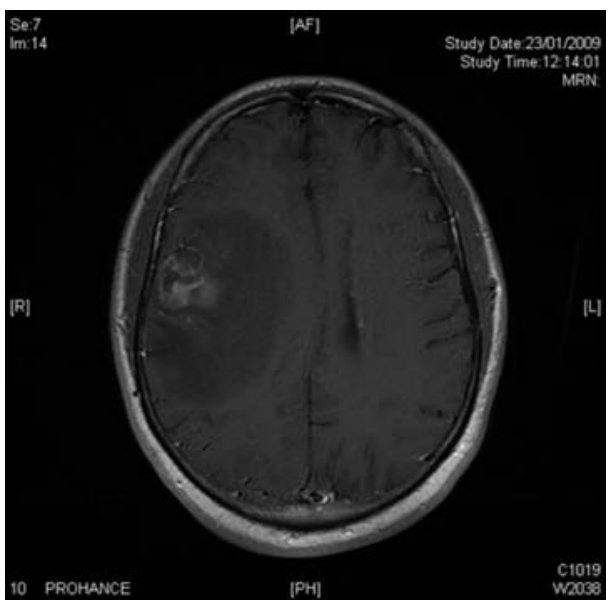

Fig 1. Cerebral toxoplasmosis.

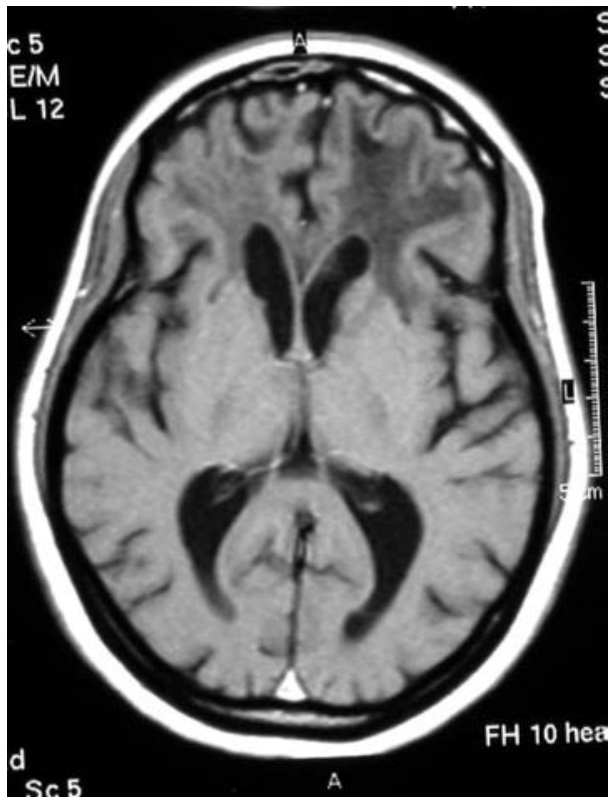

Fig 2. Progressive multifocal leukoencephalopathy.

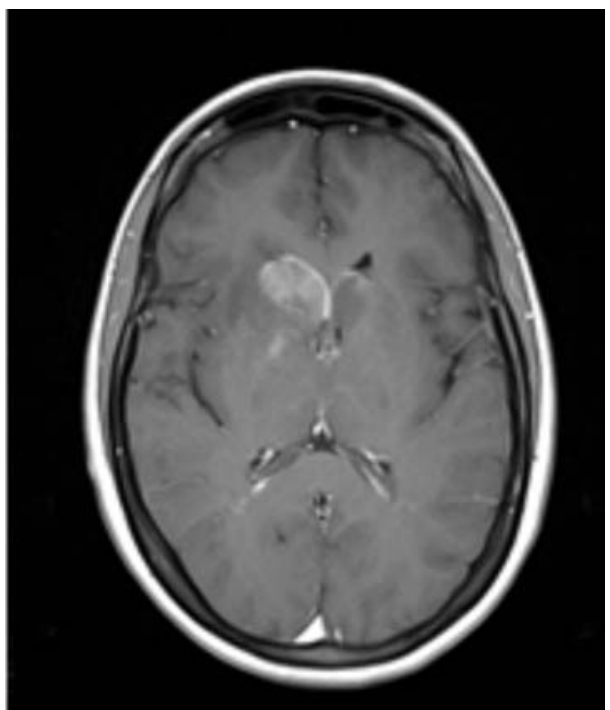

Fig 3. Primary central nervous system lymphoma. 
two weeks followed by oral high-dose fluconazole for a further eight weeks assuming the fungus is fully sensitive. cART should be optimised or initiated after the acute illness. Early commencement has possibly been associated with higher mortality.

Progressive multifocal leukoencephalopathy (PML) is an opportunistic infection caused by the John Cunningham (JC) virus and is characterised by evidence of focal demyelination. The JC virus is ubiquitous worldwide and is typically acquired in childhood with approximately $85 \%$ of all adults being seropositive. Disease occurs when CD4 counts fall, usually below $50 \mathrm{c} / \mathrm{mm}^{3}$. PML presents with slowly progressive focal neurological deficits, especially speech and visual defects, and white matter abnormalities without surrounding oedema on MRI. The diagnosis is supported by the presence of the JC virus within CSF (sensitivity $>90 \%$ if not on cART). Definitive diagnosis is with a brain biopsy but the presence of MRI and CSF findings is sufficient to make the diagnosis in all but a minority of cases. cART remains the only effective treatment. ${ }^{9,10}$

\section{HIV-related malignancy}

HIV-associated primary CNS lymphoma is typically a diffuse large (B) cell non-Hodgkin's lymphoma. Marked immunosuppression and infection with Epstein-Barr virus (EBV) are implicated in its development. The presentation is often indistinguishable from toxoplasmosis, with symptoms of headache, focal neurological deficits and seizures; the history, however, is usually sub-acute. Imaging typically reveals a single homogeneously enhancing mass lesion in the periventricular space with mild to moderate surrounding oedema. CSF reveals a slightly raised protein but only $20 \%$ of patients have malignant cells on cytology. Histological examination remains the gold standard of diagnosis and should always be obtained. However, a presumptive diagnosis can be made if there is evidence of the EBV within the CSF ( $>90 \%$ sensitivity and $>95 \%$ specificity) and the presence of a single lesion on scan that does not respond to typical toxoplasmosis treatment. ${ }^{11}$ Despite chemotherapy treatment the prognosis remains poor.

\section{Non-AIDS-related disease}

The incidence of stroke is higher in the HIV population (1-2\% of patients). The aetiology may be directly or indirectly related to HIV. Secondary causes include mass lesions, such as toxoplasmosis and primary CNS lymphoma (when vascular invasion may contribute), an increased use of illicit drugs (especially cocaine) and vasculitis caused by several opportunistic infections (eg herpes simplex encephalitis, syphilis, tuberculosis and cryptococcus). HIV has also been implicated in causing small and medium vessel vasculopathy, with dyslipidaemia and increased inflammation possibly leading to endothelial activation and dysfunction. ${ }^{12}$

Certain ARV agents, through causing mitochondrial dysfunction, have been an important cause of peripheral neuropathy with didanosine (ddI), zalcitabine ( $\mathrm{ddC}$ ) and stavudine ( $\mathrm{d} 4 \mathrm{~T})$ being the most important agents implicated. In addition, both ddI and $\mathrm{d} 4 \mathrm{~T}$ have been rarely associated with a GB-like syndrome, especially during pregnancy. However, with the withdrawal of all three drugs from first line therapy (and $\mathrm{ddC}$ completely), they are rarely used and these complications are no longer being seen outside of resource-poor nations.

\section{Summary}

HIV can present in many guises and may be associated with significant morbidity and mortality. Although cART has reduced the incidence of classic opportunistic infections and tumours seen in late-stage HIV significantly, early recognition of HIV at seroconversion or later in advanced disease remains vital. The presentation and treatment of opportunistic infections has remained relatively unchanged over the years, but the use of cART has markedly improved post-treatment prognosis. Early recognition is therefore essential as a delay in considering HIV can lead to inappropriate treatment, investigation and initiation of effective ARV therapy. HIV should therefore be considered in all acute neurological syndromes (aseptic meningitis, GB syndrome, cerebrovascular accident, mass lesions, etc) and an HIV antibody test performed urgently. In the case of primary syndromes, a prompt diagnosis may prevent severe HIV-related disease in the future.

\section{References}

1 Shapshak P, Kangueane P, Fijimura RK et al. Editorial NeuroAIDS review. AIDS 2011;25:123-41.

2 Letendre SL, Ellis RJ, Everall I et al. Neurologic complications of HIV disease and their treatment. Top HIV Med 2009;17:46-56.

3 Childs EA, Lyles RH, Selnes OA et al. Plasma viral load and CD4 lymphocytes predict HIV-associated dementia and sensory neuropathy. Neurology 1999;52:607.

4 Parsons TD, Tucker KA, Hall CD et al. Neurocognitive functioning and HAART in HIV and hepatitis $\mathrm{C}$ virus co-infection. AIDS 2006;20:1591-5.

5 Sacktor N. The epidemiology of human immunodeficiency virusassociated neurological disease in the era of highly active antiretroviral therapy. J Neurovirol 2002;8(Suppl 2):115-21.

6 Letendre S, Marquie-Beck J, Capparelli E et al; CHARTER group. Validation of the CNS penetration-effectiveness rank for quantifying antiretroviral penetration of the central nervous system. Arch Neuro 2008;65:65-70.

7 Kaplan JE, Benson C Holmes KK et al. Guidelines for prevention and treatment of opportunistic infections in HIV-infected adults and adolescents. Recommendations from CDC, the National Institutes of Health, and the HIV Medicine Association of the Infectious Diseases Society of America. MMWR Recomm Rep 2009;58:1-207.

8 Portegies P, Solod L, Cinque P et al. Guidelines for the diagnosis and management of neurological complications of HIV infection. Euro J Neurol 2004;11:297-304.

9 Mirrales P, Berenguer J, Garcia de viedma D et al. treatment of AIDS associated progressive multifocal leukoencephalopathy with highly active antiretroviral therapy. AIDS 1998;12:2467-72.

10 Clifford DB, Yiannoutsos C, Glicksman M et al. HAART improves prognosis in HIV-associated progressive multifocal leukoencephalopathy. Neurology 1999;52:623-5.

11 Arribas JR, Clifford DB, Fichtenbaum CJ et al. Detection of Ebstein-Barr virus DNA in cerebrospinal fluid for diagnosis of AIDS-related central nervous system lymphoma. J Clin Microbiol $1998 ; 33: 1580-3$ 
12 Dobbs MR, Berber JR. Stroke in HIV infection and AIDS. Expert Rev Cardiovasc Ther 2009;7:1263-71.

Address for correspondence: Dr C Hogan,

Department of Infectious Diseases,

North Manchester General Hospital,

Delaunays Road, Crumpsall, Manchester M8 5RB.

Email: celiahogan@hotmail.com

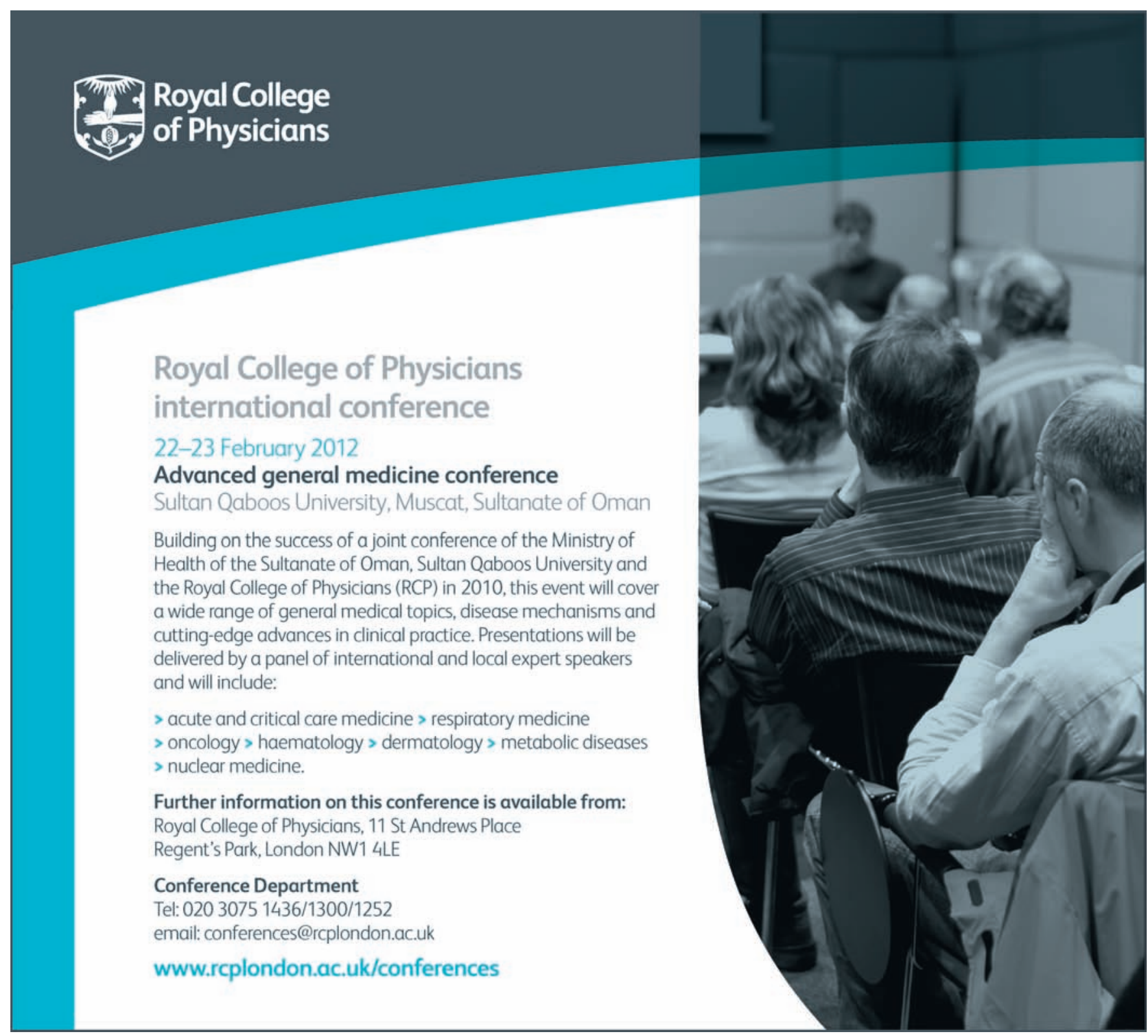

